\title{
A Simple Algorithm for Image Denoising Based on MS Segmentation
}

\author{
G. Vijaya ${ }^{1}$ \\ Senior Lecturer, Dept. of CSE, \\ Kalasalingam University, \\ Krishnankoil , Tamilnadu, India.
}

\author{
Dr.V.Vasudevan ${ }^{2}$ \\ Senior Prof. \& Head Dept. of IT, \\ Kalasalingam University, \\ Krishnakoil, Tamilnadu, India.
}

\begin{abstract}
Image Denoising \& Segmentation are the key issues in all image processing researchers. The first step in image processing is segmentation. This can be done by using MS (Mean Shift) segmentation. After segmentation the image, the overall system quality can be improved by using the bilateral filter. The proposed method improves the bilateral filter through decomposing a signal into its frequency components. In this way, noise in different frequency component can be eliminated. Experimental results shown that our algorithm outperforms the other algorithms.
\end{abstract}

Keywords: image Denoising, segmentation, Mean Shift (MS), bilateral filter.

\section{INTRODUCTION}

Image Denoising is one of the important steps in image preprocessing for performing high - level vision tasks such as recognition and scene interpretation. The goal of image Denoising is to remove noise while keeping the edges, corners and texture details as much as possible. A noisy image can be represented as,

$$
\mathrm{v}(\mathrm{i})=\mathrm{u}(\mathrm{i})+\mathrm{n}(\mathrm{i}) \text {, }
$$

where $v(i)$ is the observed value, $u(i)$ is the true value and $\mathrm{n}(\mathrm{i})$ is the noise value at the pixel i. One common type of noise is Gaussian noise. This type of noise contains variations in intensity that are drawn from a Gaussian distribution and is a very good model for many kind of sensor noise.

Ideally, the same image model should be used for both noise estimation and Denoising. We found that segmentation - based approach is well suited to both tasks. After a natural image is segmented into smooth regions, the pixel value within each segment approximately lie on a 1D line in RGB space due to the physics of image formation [4],[5],[6]. This important fact can help to significantly reduce color noise. We further improve the results by applying Bilateral Filtering to clean image.

\section{RELATED WORK}

In this section, we briefly review previous work on image Denoising and segmentation.

\subsection{Image Denoising}

Several methods have been proposed to remove noise and try to recover the "true" image. One simple form to remove noise is by convolving the original image with a mask that represent a low-pass filter (eg. Gaussian filter), performing a smoothing operation through a neighborhood weighted averaging, in which, the weights decrease with distance from the centered pixels. Frequently, employing smoothing filters present significant edge blurring.

Non - linear filters are widely used to reject noise methods based on neighborhood ranking (eg. Median filter) are classic examples; some of these methods cause relatively little blurring of edges.

Another method for removing noise is to evolve the image under a smoothing partial differential equation similar to the heat equation which is called anisotropic diffusion [7].

Recently, bilateral filtering [8] has become a popular method that provides very good results for image Denoising. This proposal combines pixel values based in geometric closeness and intensity similarity, preferring near values to distant values and averaging image intensity values with weights that decay with dissimilarity.

\subsection{Color Image Segmentation}

Image Segmentation is a process that partitions an image into different regions such that each region is nearly homogeneous, whereas the union of any two regions is not. It serves as a key in image analysis and pattern recognition and is a fundamental step in low-level vision, which is significant for object - recognition and tracking, image retrieval, face detection and other computer - vision related applications [10]. 
Existing image segmentation algorithm can be generally classified into three major categories, i.e., feature-spacebased clustering, spatial segmentation and graph-based partitioning. Feature-space-based clustering approaches [11],[12] capture the global characteristics of the image through the selection and calculation of the image features, which are usually based on the color or texture. Although the data clustering approaches are efficient in finding salient image features, they have some serious drawbacks as well. The spatial structure and the detailed edge information of the image are not preserved, and pixels from disconnected regions of an image may be group together if the feature space is overlaps. The spatial segmentation method is also referred as region-based when it is based on region entities. The watershed algorithm [13] is an extensively used technique for this purpose.

Graph - based approaches [14] can be regarded as image perceptual grouping and organizational methods based on the fusion of the future and spatial information. The common theme underlying these approaches is the formation of a weighted graph, where each vertex corresponds to a image pixel, and the weight of each edge connecting two pixels represent the likelihood they belongs to the same segment. The weights are usually related to the color and texture features, as well as the spatial characteristics of the corresponding pixels or regions. The overall segmentation performance of the graph partitioning approach is sensitive to the region segmentation results and the graph grouping strategy.

To overcome these problems, we propose in this correspondence a novel approach that provides effective and robust image segmentation with low computational complexity by incorporating the Mean Shift (MS) method [15].

\section{MEAN SHIFT (MS) SEGMENTATION}

The MS algorithm is a robust feature - space analysis approach which can be applied to discontinuity preserving smoothing and image segmentation problems. It can significantly reduce the number of basic image entities, and due to the good discontinuity preserving filtering characteristic, the salient features of the overall image are retained. The later property is particularly important in the portioning of natural images, in which only several distinct regions are used in representing different scenes such as sky, lake, sand beach, person and animal, whereas other information within a region is often less important and can be neglected. MS algorithm is an unsupervised clustering based segmentation method, where the number and the shape of the data cluster are unknown, a priori. Moreover, the termination of the segmentation process is based on some region - merging strategy applied to the filtered image result, and the number of regions in the segmented image is mainly determined by the number of pixels in a region which is denoted as $\mathrm{M}$ (i.e., regions containing less than $\mathrm{M}$ pixels will be eliminated and merged into its neighboring region).

In this section, we present a brief review of the image segmentation method based on the MS procedure. We consider radially symmetric kernels satisfying $\mathrm{K}(\mathrm{x})=$ $c_{k, d} k\left(\|x\|^{2}\right)$, where constant $c_{\mathrm{k}, \mathrm{d}}>0$ is chosen such that $\int_{0}^{\infty} K^{\prime \prime}(x) d x=\int_{0}^{\infty} c_{k, d} k\left(\|x\|^{2}\right) d x=1$ [note that $\mathrm{k}(\mathrm{x})$ is defined only for $\mathrm{x} \geq 0]$. $\mathrm{K}(\mathrm{x})$ is a monotonically decreasing function and is referred to as the profile of the kernel. Given the function $\mathrm{g}(\mathrm{x})=-\mathrm{k}^{\prime}(\mathrm{x})$ for profile, the kernel $\mathrm{G}(\mathrm{x})$ is defined as $G(x)=c_{g, d} g\left(\|x\|^{2}\right)$. For $\mathrm{n}$ data points $x_{I}, i=1 \ldots n$ in the d-dimensional space $R^{d}$, the MS is defined as,

$$
m_{h, G}(x)=\frac{\sum_{i=1}^{n} x_{i} g\left(\left\|\frac{x-x_{i}}{h}\right\|^{2}\right)}{\sum_{i=1}^{n} g\left(\left\|\frac{x-x_{1}}{h}\right\|^{2}\right)}-x
$$

Where $\mathrm{x}$ is the center of the kernel (window), and $\mathrm{h}$ is a bandwidth parameter. Therefore, the MS is the difference between the weighted mean, using kernel $\mathrm{G}$ as the weights and $\mathrm{x}$ as the center of the kernel (window). Regions of lowdensity values are of no interest for the feature - space analysis, and in such regions, the MS steps are large. On the other hand, near local maxima, the steps are small, and the analysis is more refined. The MS procedure, thus, is an adaptive gradient ascent method [16]. The central position of kernel $G$ can be updated iteratively by,

$$
y_{j+1}=\frac{\sum_{i=1}^{n} x_{i} g\left(\left\|\frac{y_{j}-x_{i}}{h}\right\|^{2}\right)}{\sum_{i=1}^{n} g\left(\left\|\frac{y_{j}-x_{i}}{h}\right\|^{2}\right)}, \quad j=1,2, \ldots
$$

Where $y 1$ is the center of the initial position of the kernel. Based on the above analysis, the MS segmentation algorithm can be obtained and this is shown in Fig.1. 


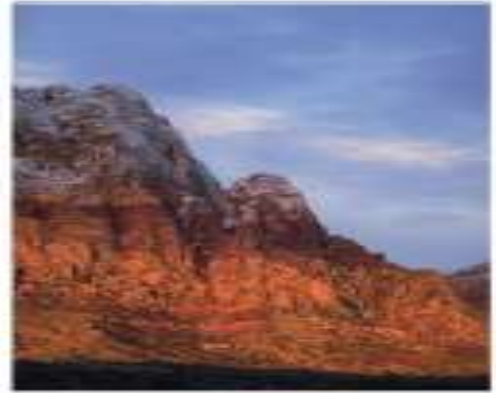

(a)

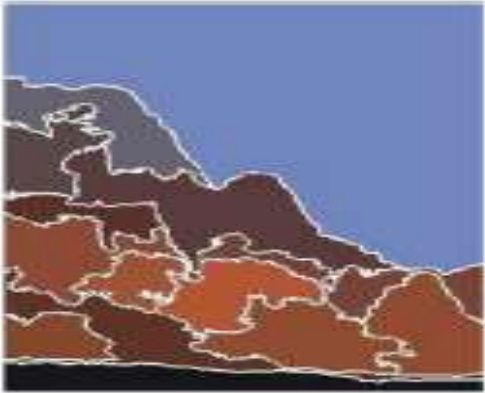

(b)

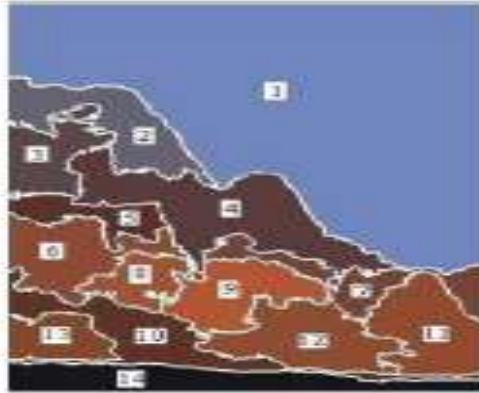

(c)

Fig. 1. a) Original Image. b) Result image after using MS segmentation algorithm. c) Labeled Regions.

\section{BILATERAL FILTERING}

The bilateral filter [3] was alternative to wavelet thresholding. It applies spatial weighted averaging without smoothing edges. This is achieved by combining two Gaussian filters: One filter works in spatial domain and the other filter works in intensity domain. Therefore, not only the spatial distance but also the intensity distance is important for the determination of weights. At a pixel location $\mathrm{x}$, the output of a bilateral filter can be formulated as follows:

$$
\tilde{I}(\mathrm{x})=\frac{1}{C} \sum_{\mathrm{y} \in \mathcal{N}(\mathrm{x})} e^{\frac{-\|\mathrm{y}-\mathrm{x}\|^{2}}{2 \sigma_{d}^{2}}} e^{\frac{-\mid I(\mathrm{y})-I(\mathrm{x}) \|^{2}}{2 \sigma_{r}^{2}}} I(\mathrm{y})
$$

Where $\sigma_{\mathrm{d}}$ and $\sigma_{\mathrm{r}}$ are parameters controlling the fall-off of weights in spatial and intensity domains, $\mathbb{N}(x)$ is a spatial neighborhood of pixel $\mathrm{I}(\mathrm{x})$, and $\mathrm{C}$ is the normalization constant:

$$
C=\sum_{\mathbf{y} \in \mathcal{N}(\mathbf{x})} e^{\frac{-\|\mathbf{y}-\mathbf{x}\|^{2}}{2 \sigma_{\mathrm{d}}^{2}}} e^{\frac{-\| I(\mathbf{y})-\left.I(\mathbf{x})\right|^{2}}{2 \sigma_{T}^{2}}} .
$$

There are two parameter that control the behavior of the bilateral filter. Referring to (2), $\sigma_{\mathrm{d}}$ and $\sigma_{\mathrm{r}}$ characterizes the spatial and intensity domain behavior respectively. Although, these parameters should be related to the noise and image characteristics, the issue has not been studied yet. In this paper, we made an empirical study of the optimal parameter values as a function of noise variance. We added white Gaussian noise to some standard images and applied the bilateral filter for different values of the parameters $\sigma_{\mathrm{d}}$ and $\sigma_{\mathrm{r}}$. We repeated the experiment for different noise variances and record the mean squared error (MSE). A typical mean squared error (MSE) plot is given in Fig. 2
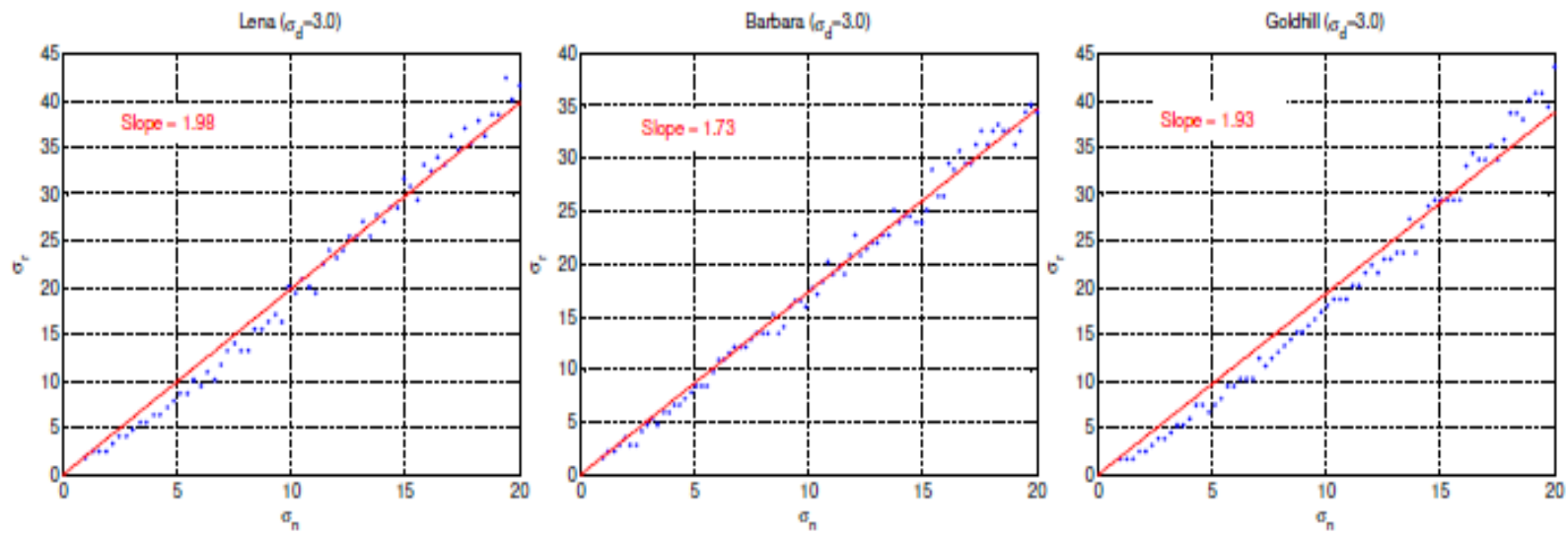

Fig. 2. The optimal $\sigma_{\mathrm{r} \text {. }}$ as a function of noise standard deviation $\sigma_{\mathrm{d}}$ are plotted for three standard images. The image sizes are 512 $\mathrm{x} 512$. 


\section{SEGMENTATION BASED DENOISING}

Mean Shift (MS) algorithm is a powerful technique for image segmentation. The mean shift vector always point toward the direction of the maximum increase in the density. The mean shift procedure, obtained by successive,

- $\quad$ Computation of the mean shift vector $m_{h},\left(x^{t}\right)$.

- Translation of the window $x^{t+1}=x^{t}+m_{h},\left(x^{t}\right)$.

Is guaranteed to converge to a point where the gradient of density function is zero.

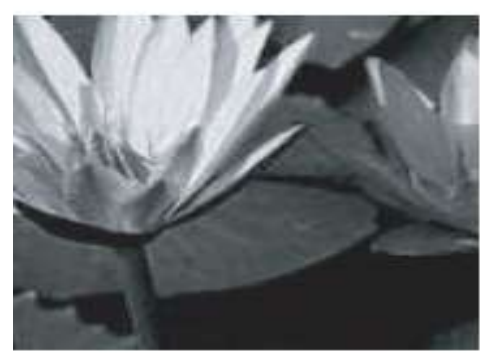

(a)

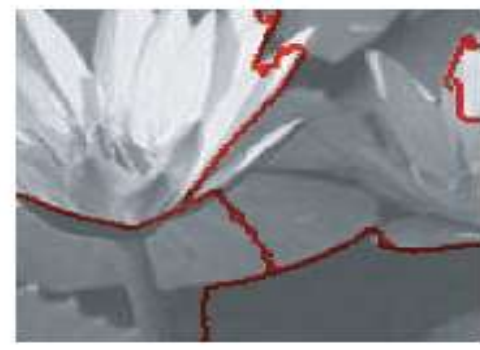

(b)
The mean shift clustering algorithm is[Type a quote from the document or the summary of an interesting point. You can position the text box anywhere in the document. Use the Text Box Tools tab to change the formatting of the pull quote text box.]

In order to obtain a more fair runtime comparison of mean shift and normalized cuts, we tested the two methods on very small gray scale images that the normalized cut code does not need to modify. Table 1 shows the runtimes for the segmentation results Fig.4 \& Fig. 5. In this experiment both methods found a segmentation on an image with the same number of pixels. We see that even when $\mathrm{h}$ is large, mean shift is significantly faster than normalized cut.

Fig.3. The lily pad image: 147 x 135 pixels. (a) Original Image, (b) Segmentation obtained by Normalized Cut \& (c) Segmentation obtained by Mean Shift (MS) using $h=[20,20]$ and $M=20$.

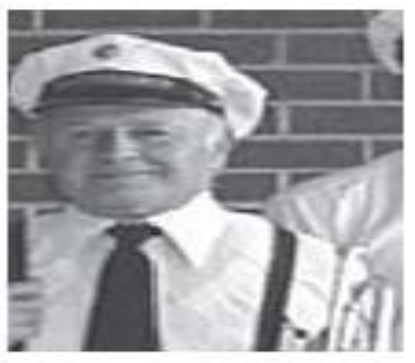

(a)

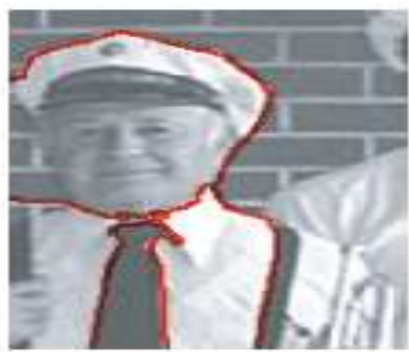

(b)

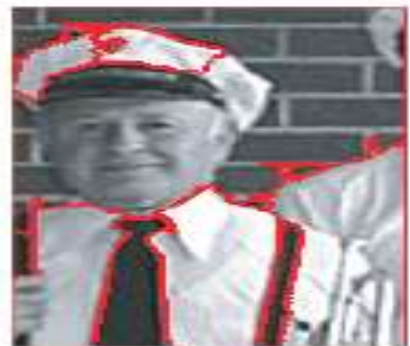

(c)

Fig. 4. The trumpet image: 118 x 166 pixels. (a) The input image, (b) The segmentation obtained by Normalized Cuts \& (c) The segmentation obtained by Mean Shift (MS) using $\mathrm{h}=[20,20]$ and $\mathrm{M}=20$.

\begin{tabular}{|c|c|c|}
\hline Method & Lily Pad & Trumpet \\
\hline Normalized Cuts & 10.1 & 10.9 \\
Mean Shift & 1.53 & 1.37 \\
\hline
\end{tabular}

Table.1. A comparison of the runtime between the normalized cut and mean shift methods on small images.

After the image has undergone preprocessing steps segmenting the image, by using MS clustering segmentation, we go for Denoising. This can be illustrated in Fig.6. 


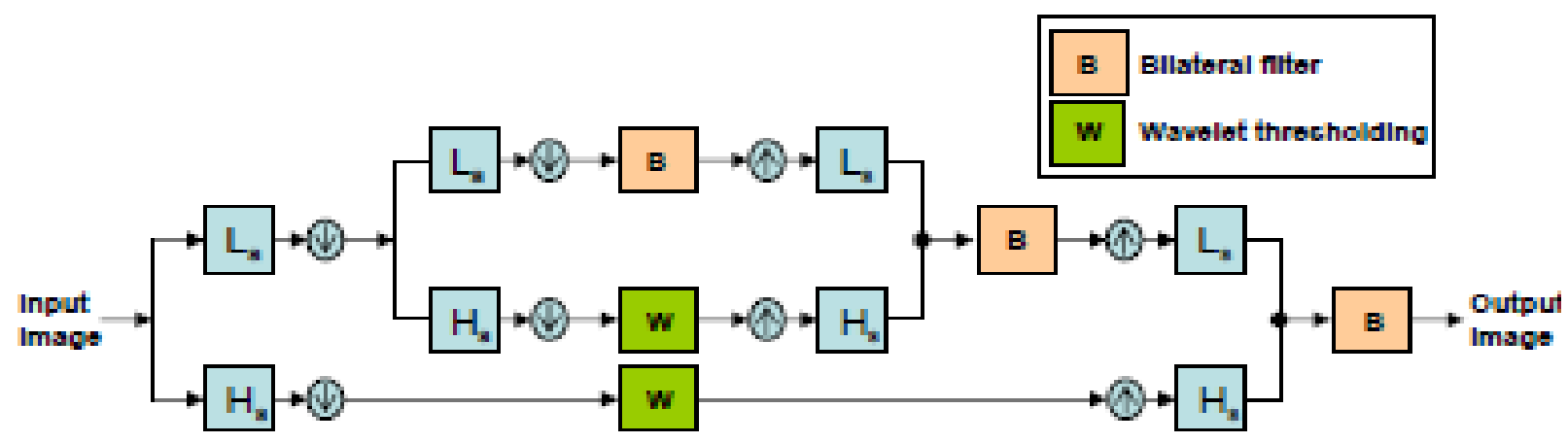

Fig. 5. Illustration of the proposed method. Image is decomposed into its low- and high-frequency components through analysis filters. Bilateral filter is applied to low-frequency components; wavelet thresholding is applied to high-frequency components.

In the illustration, two types of filtering are applied on the image. The first one is the bilateral filtering, which is applied to the image and its approximation subbands. The second is the wavelet thresholding, which is applied to the detail subbands of the image. The multi - resolution bilateral filtering is a generalization of standard bilateral filtering. Bilateral filtering works in approximation subbands; however, some noise components can be identified and removed better in detail subbands, for example, salt-and-pepper type of noise. The wavelet thresholding part is optional but it provides this additional capability to the frame work. There are different ways of estimating the noise levels in images and in different subbands of an image. In our experiments, we used robust median estimator [ 17] to estimate noise variance. The method fits the proposed frame work well as it is also wavelet based. For the wavelet thresholding, we used the Bayes Shrink soft thresholding method, which utilizes the same robust median estimator to determine threshold values.

\section{EXPERIMENTAL RESULTS}

These images were denoised using four methods. The first method is the wavelet thresholding algorithm [9]. Five decomposition levels were used; the noise variance is estimated using the robust median estimator [16]. The second method is the bilateral filter [3]. Based on our experiments discussed in the previous section, we choose the following parameter for the bilateral filter: $\sigma_{\mathrm{d}}=1.8, \sigma_{\mathrm{r}}=$ $2 \mathrm{x} \sigma_{\mathrm{n}}$, and the window size is $11 \mathrm{x} 11$. The third method is the sequential application of [9] and [3]. The fourth method is the proposed method. For the proposed method, db8 filters in Matlab[2] were used for one-level decomposition. For the Bilateral filtering part of the proposed method, we set the parameters as follows: : $\sigma_{\mathrm{d}}=1.8$, the window size is $11 \times 11$, and $\sigma_{\mathrm{r}}=1.0 \times \sigma_{\mathrm{n}}$, at each level. (In case of the original Bilateral filter, $\sigma_{\mathrm{r}}=2 \times \sigma_{\mathrm{n}}$ was a better choice. However, for the proposed method, this lead to a smaller PSNR value on the average. The reason is the double application of the bilateral filter in the proposed method. When $\sigma_{\mathrm{r}}$ was large, texture in the images, was smoothed to produce low PSNR values. After some experimentation, $\sigma_{\mathrm{r}}$ $=1.0 \times \sigma_{\mathrm{n}}$ turned out to be better in terms of PSNR values. Here, we should note the fact that a higher PSNR does not necessarily correspond to a better visual quality). For the wavelet thresholding part of the proposed method, the Bayes Shrink method [9] was used; and the noise variance was estimated again with the robust median estimator technique. To eliminate the border effects, images were mirror-indexed before the application of the bilateral filter and cropped to the original size at the end. The PSNR results are given in Table2.
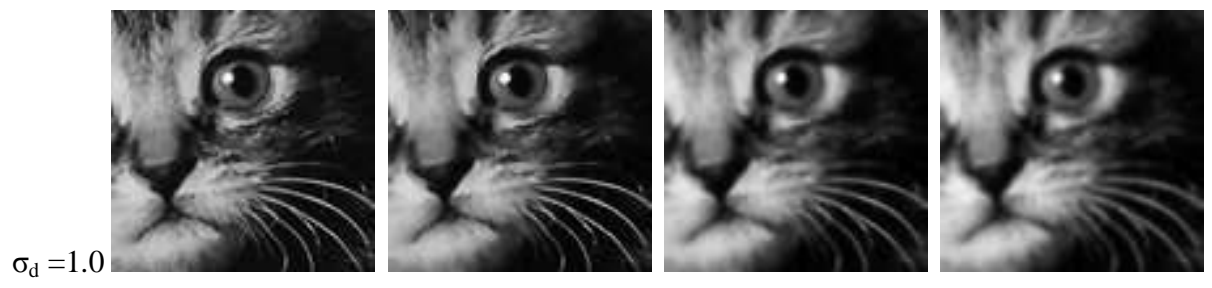

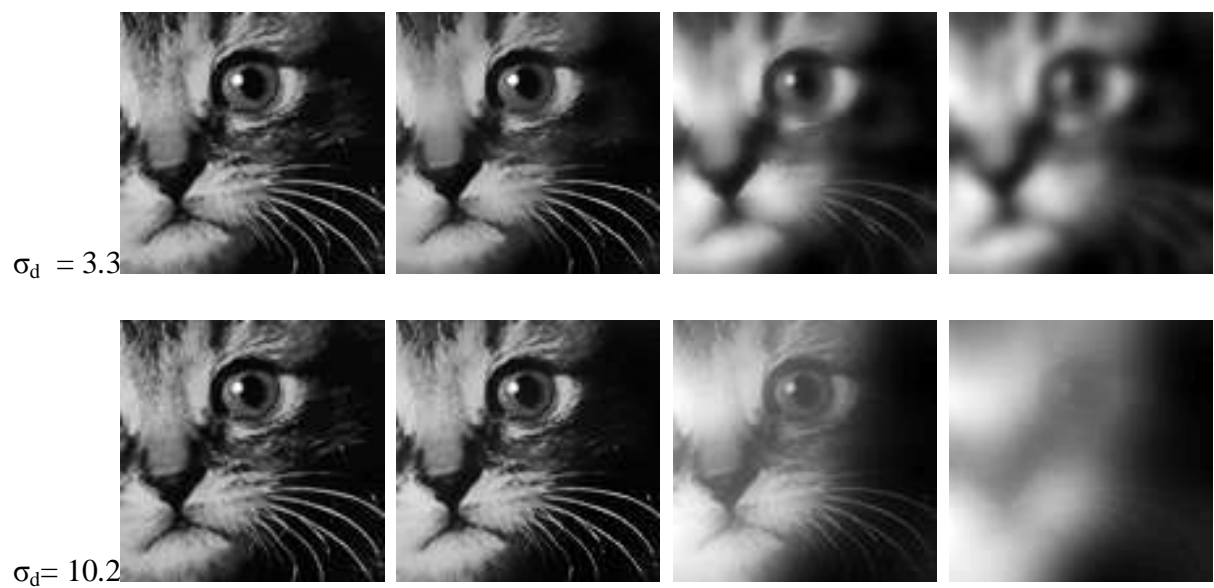

$\sigma_{\mathrm{r} .}=10$

$\sigma_{\mathrm{r} .}=30$
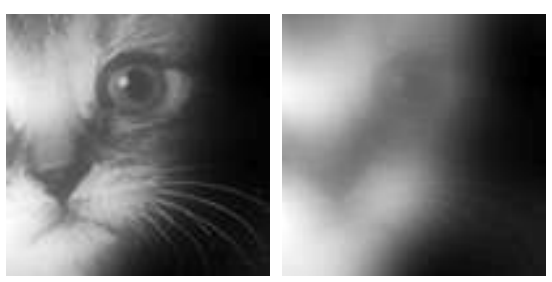

$\sigma_{\text {r. }}=100 \sigma_{\text {r. }}=300$

Fig. 6. Process with bilateral filters with various range and domain parameter values.

\begin{tabular}{|c||c|c|c|c|c|}
\hline Input Image & $\sigma_{n}$ & BayesShrink [9] & Bilateral Filter [3] & [9] Followed by [3] & Proposed Method \\
\hline Barbara 512 5512 & 10 & 31.25 & 31.37 & 30.92 & 31.79 \\
& 20 & 27.32 & 27.02 & 27.16 & 27.74 \\
& 30 & 25.34 & 24.69 & 25.23 & 25.61 \\
\hline Boats 512 5512 & 10 & 31.98 & 32.02 & 31.81 & 32.58 \\
& 20 & 28.55 & 28.40 & 28.43 & 29.25 \\
& 30 & 26.71 & 26.57 & 26.66 & 27.24 \\
\hline Goldhill 512 5512 & 10 & 31.94 & 32.08 & 31.93 & 32.48 \\
& 20 & 28.69 & 28.90 & 28.80 & 29.50 \\
& 30 & 27.13 & 27.50 & 27.34 & 27.77 \\
\hline
\end{tabular}

Table 2. PSNR comparison of the Bayes Shrink method [9], the bilateral filter [3], sequential applications of Bayes Shrink [9] and Bilateral filter [3] and the proposed method. (The numbers are obtained by averaging the results of six runs).

\section{CONCLUSION}

In this paper a new segmentation based image Denoising technique was followed. The proposed method takes the advantage of the MS segmentation method, whereas their drawbacks are avoided. After segmenting the image using MS method, we perform Denoising by integrating the bilateral filter and wavelet thresholding together. We decompose the image into low- and high-frequency components, and apply bilateral filtering on the approximation subbands and wavelet thresholding on the detail subbands. We have found that the optimal value $\sigma_{\mathrm{r}}$ value of the bilateral filter is linearly related to the standard deviation of the noise. The optimal value of the $\sigma_{d}$ is relatively independent of the noise power. Based on these results, we estimate the noise variance at each level of the subband decomposition and use the optimal $\sigma_{\mathrm{r}}$ value for the bilateral filtering. Experiments were conducted for the proposed method which outperforms the state-of-art existing image Denoising algorithms.

\section{REFERENCES}

[1].Rafael C.Gonzalez and Richard E.Woods, "Digital Image Processing", Third Edition, PHI Learning Private Ltd., 2008.

[2]. Duane Hanselman, and Bruce Littlefield, "Mastering MATLAB 7”, Pearson Education, 2005.

[3]. C. Tomasi and R. Manduchi, "Bilateral filtering for gray and color images," in Proc. Int. Conf. Computer Vision, 1998, pp. 839-846.

[4]. T.Gevers and A.W.M. Smeulders, "Color - Based Object Recognition”, Pattern Recognition, vol.32, no.3, pp. 453 - 464, 1999. 
[5]. G.Healey,"Segmenting Images Using Normalized Color", IEEE Trans. Systems, Man, and Cybernetics, vol. 26, no.10, pp. 1272 - 1282, Oct. 2004.

[6]. G.Klinker, S.Shafer and T.Kanade, "A Physical Approach To Color Image Understanding”, Int'l. Jr. Computer Vision, vol.4, no.1, pp. 7 - 38, Jan. 1990.

[7]. P.Perona and S.Malik, "Scale - Space and Edge Detection Using Anisotropic Diffusion", IEEE Trans. On Pattern Analysis and Machine Intelligence, 1990, vol. 12, no.7, pp. $629-639$.

[8]. Ming Zhang and Bahadir Gunturk, "A New Image Denoising Method Based on the Bilateral Filter", IEEE Intl. Conference on Acoustics, Speech and Signal Processing, May 2008.

[9]. S. G. Chang, B. Yu, and M. Vetterli, "Adaptive wavelet thresholding for image denoising and compression," Trans. ImageProcessing, vol. 9, no. 9, pp. 1532-1546, September 2000.

[10]. N.Pal and S.Pal,"A Review on Image Segmentation Techniques", Pattern Recognition, vol. 26, no. 9, pp. 1277 - 1294, Sep. 1993.

[11]. D.W.Jacobs , D.Weinshall and Y.Gdalyahu, "Classification with non-metric distances: Image Retrieval and Class Representation,",
IEEE Trans. Pattern Annal. Mach.Intell., vol. 22, no. 6, pp. $583-600$, Jun. 2000.

[12]. A.K.Jain and D.Zongker, "Representation and recognition of handwritten digits using deformable templates", IEEE Trans. Pattern Annal. Mach.Intell., vol. 19, no. 12, pp. 1386 - 1391, Dec. 1997.

[13]. L. Vincent and P. Soille, "Watersheds in digital spaces: An efficient algorithm based on immersion simulation," IEEE Trans. Pattern Anal. Mach. Intell., vol. 13, no. 6, pp. 583-597, Jun. 1991.

[14]. P.F.Felzenszwalb and D.P.Huttenlocher, "Efficient Graph - Based Image Segmentation", Int'l. J. Computer Vision, vol. 59, pp. 167 - 181, 2004.

[15] .D. Comaniciu and P. Meer, "Mean shift: A robust approach toward feature space analysis," IEEE Trans. Pattern Anal. Mach. Intell., vol. 24, no. 5, pp. 603-619, May 2002.

[16]. R. van den Boomgaard and J. van de Weijer, "On the equivalence of local-mode finding, robust estimation and mean-shift analysis as used in early vision tasks," in Proc. Int Conf. Pattern Recog., 2002, pp. 30 927-30930.

[17]. D. L. Donoho and I. M. Johnstone, "Ideal spatial adaptation by wavelet shrinkage," Biometrika, vol. 81, no. 3, pp. 425-455, 1994. 